\title{
Improvement of Collagen Gel/Sponge Composite Scaffold by Gel Wrapping for Cartilage Tissue Engineering
}

\author{
Yusuke Nakamuta1 ${ }^{1}$, Mitsugu Todo ${ }^{2}$, Takaaki Arahira ${ }^{3}$ \\ ${ }^{1}$ Kyushu University, 6-1 Kasuga-Koen, Kasuga, Japan. \\ 2 Research Institute for Applied Mechanics, Kyushu University, 6-1 Kasuga-Koen, Kasuga. \\ 3 Fukuoka Dental College, 2-15-1 Tamura, Sawara-ku, Fukuoka. \\ * Corresponding author. Tel.: 092-583-7762; email: todo@riam.kyushu-u.ac.jp \\ Manuscript submitted October 9, 2016; accepted February 3, 2017. \\ doi: 10.17706/ijbbb.2017.7.2.102-109
}

\begin{abstract}
Tissue engineering approach for cartilage regeneration has clinically been conducted using chondrocytes with collagen gel scaffold. However, such engineered cartilage tissue cannot be implanted into the load-bearing part of joint surface because of poor mechanical properties that are much lower than those of natural cartilage tissue. The aim of this research project is, therefore, to develop a novel engineered cartilage tissue that has mechanical properties comparable with natural articular tissue. As a new novel scaffold for cartilage regeneration, collagen gel/sponge composite scaffold was developed by placing sponge structure in gel phase. It was found that the composite scaffold has much better compressive mechanical properties than pure collagen gel. Human mesenchymal stem cells (hMSCs) were also cultured with a standard cell growth medium, and nicely proliferated in the scaffold. Next, combined culture condition such as the condition: chondrogenic differentiation and growth mediums, were examined. It was found that conditions can effectively activate the proliferation and differentiation of MSCs, resulting in increase of compressive property. As the next step, Improvement of the structure of the composite scaffold were examined. Many chondrocyte-like spherical cells and many collagen fibrils were observed on the surface of the samples.
\end{abstract}

Key words: Tissue engineering, mesenchymal stem cell, composite material, biopolymer.

\section{Introduction}

When the cartilage is damaged by osteoarthritis or acetabular dysplasia, the joint function is usually lost with severe pain. Damaged cartilage cannot be recovered due to luck of circulation system. Until now, artificial joint replacement is the only effective treatment for the last stage of osteoarthritis [1]. However, recently, clinical applications of tissue engineered cartilage regeneration have been studied for the damaged cartilage. Some approaches for the defect site regeneration have been conducted by transplanting engineered cartilages to the damaged portion [2], [3]. For example, Adachi et al. investigated clinical application using a tissue-engineered cartilage-like tissue that was prepared by culturing autologous chondrocytes in atelocollagen gel for a certain period [2]. Also, Liao et al. examined a tissue-engineered cartilage that was produced by culturing chondrocytes in porous scaffold [3].

The autologous chondrocytes have been used basically as the cell source for the production of tissue-engineered cartilage. It provides excellent biocompatibility without inducing host immune response. However, it causes a new damage by collecting cartilage tissue from healthy point of the patient. Therefore, 
mesenchymal stem cells (hMSCs) and iPS cells are expected as a new source of cells [4]. HMSCs harvested from patient do not induce host immune responses. It also has advantage such as multilineage potential and can be abundantly available collected from bone marrow and lipid of patient [5], [6]. However, at the present stage, the difficulty of the differentiation potential control of stem cells that have the properties to differentiate into a variety of cells such as osteoblasts and chondrocytes, and long-term cell culture and tissue construct in vitro have become problems. As an example of application to cartilage regeneration, research of the development of tissue engineered cartilage using MSC and a hydrogel [7], and research of differentiation of iPS cells into chondrocytes [8] have been carried out.

Selection of the scaffold is also important in the development of tissue engineered cartilage. Until now, atelocollagen gel [2], [9] or a porous scaffold [10] have mainly been used. However, sufficient mechanical properties cannot be obtained in the gel alone, on the other hand, there is concern over the dedifferentiation due to the fact that the chondrocytes adhere to the scaffold surface of the porous scaffold [11]. Also, in the previous research, cell affinity in vitro of the scaffold, or the cartilage tissue forming ability of the in vivo by animal experiments have mainly been focused, and researches conducted from the mechanical point of view are very limited. In particular, a few studies that investigated in detail the effects of cell proliferation and differentiation and extracellular matrix formation (ECM) on the mechanical properties of scaffolds in vitro have been performed [12]-[14]. For example, Yodmuang et al. examined the equilibrium modulus of the tissue-engineered cartilage made with silk fiber-hydrogel composites and chondrocytes [14].

In previous research, we have prepared a new collagen gel/sponge composite scaffold (Fig. 1 (c)) that was a composite structure of type I collagen gel (Fig. 1 (a)) and type II collagen sponge (Fig. 1 (b)) for cartilage regeneration. HMSCs used as the cell source were cultured using the composite scaffold up to 28 days under newly developed combination culture condition. It was found that such condition can effectively activate the proliferation and differentiation of hMSCs, resulting in increase of compressive property. However, many fibroblast-like flat cells were observed on the surface of the samples after 28 days combination culture.

In the present study, an innovative composite scaffold was proposed to improve the chondrogenic differentiation of hMSCs. Compressive mechanical properties, cell viability and chondrogenic marker were measured. The micro-structures of the scaffold with cells were also observed using a scanning electron microscope. Effects of the structural improvement were then discussed on the basis of the experimental results.

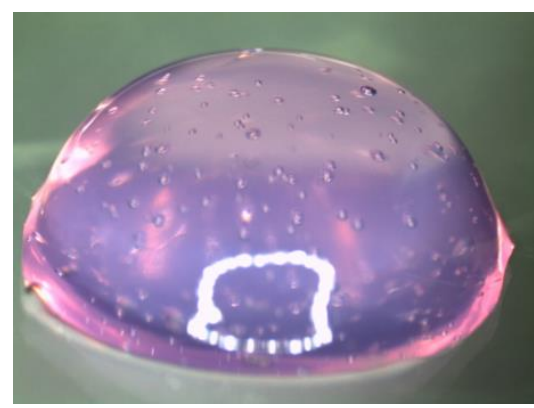

(a) Gel

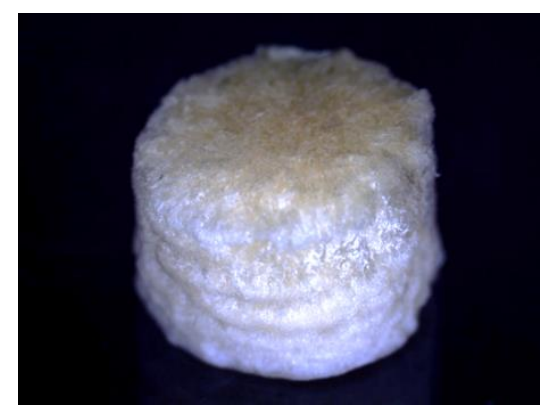

(b) Sponge

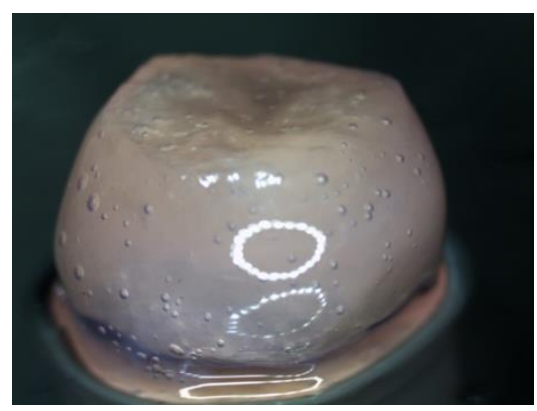

(c) Gel/sponge

Fig. 1. Over view of three different types of collagen based scaffolds.

\section{Materials and Methods}

\subsection{Scaffold Preparation}


Collagen sponges were fabricated by the freeze-drying method using chicken cartilage collagen solution (type II collagen, Nippon Meat Packers Inc.) [15]. Firstly, a silicon rubber mold was placed on a glass plate and they were wrapped in plastic wrap and pre-frozen at $-80^{\circ} \mathrm{C}$ for 1 hour. Then, the collagen solution was filled into the silicon rubber mold and frozen in the freezer again for 1 hour. After trimming the surface, it was lyophilized in an atmosphere of $-50^{\circ} \mathrm{C}$ under a vacuum for 24 hours using a freeze-dryer (EYELAFDU-1200,Tokyo Rikakikai Co.) to allow the formation of collagen sponges. The samples after lyophilization were further cross linked by treatment with glutaraldehyde vapor saturated with $25 \%$ glutaraldehyde aqueous solution (Wako Pure Chemical Industries.) at $37^{\circ} \mathrm{C}$ for $4 \mathrm{~h}$. After being cross-linked, the samples were treated with $0.1 \mathrm{M}$ of glycine aqueous solution to block unreacted aldehyde groups, and washed with deionized water and lyophilized. The diameter and thickness of the collagen sponge were about $6.5 \mathrm{~mm}$ and $5.0 \mathrm{~mm}$, respectively.

\subsection{Cell Experiment}

Gel/sponge composite scaffolds with hMSCs were produced by immersing the collagen sponge into collagen gel solution containing hMSC cells and solidifying for 1 hour in an incubator $\left(37^{\circ} \mathrm{C}, 5 \% \mathrm{CO}_{2}\right)$. Next, the new composite scaffold covered by a gel layer was produced by immersing the gel/sponge composite scaffold into the collagen gel solution and solidifying for 1 hour in an incubator. The diameter and thickness of the composite scaffold with gel layer were about $7.0 \mathrm{~mm}$ and $5.5 \mathrm{~mm}$, respectively. The type I collagen gel solution was produced by mixing Cell matrix Type I-A (Nitta Gelatin Inc.), $10 \mathrm{X}$ concentrated MEM- $\alpha$ (Nitta Gelatin Inc.), and buffer solution (Nitta Gelatin Inc.). The mixing ratio was selected as 8:1:1.1.0 × 105 $\mathrm{hMSCs}$ (Human bone marrow-derived mesenchymal stem cells, hTERT, HPV E6, expressing the E7, immortalized cell strain, cell name : UE6E7T-3, distribution source : RIKEN Bio Resource Center) were seeded per one scaffold.

Then, the composite scaffolds with gel layer containing hMSC were transferred into 24-well plates, and the cells were cultured under the combination culture condition. Firstly, 14 days culture was conducted using the cartilage differentiation medium (RPMI1640, FBS, ascorbic acid, amino acids, and antibiotics, COSMO BIO co., ltd)., and next 14 days culture was performed using the chondrocyte growth medium (RPMI1640, FBS, and antibiotics, COSMO BIO co., Itd). One sample with $1 \mathrm{ml}$ medium were placed in one well. The medium was exchanged twice a week.

\subsection{Biological Characterization}

The cell number and chondrogenic differentiation markers were measured on a regular basis. The Cell Counting Kit (Dojindo Molecular Technologies, Inc.) was used in order to measure the cell number. Firstly, samples were washed with phosphate buffered saline (PBS) to remove the media, transferred to a tube, immersed in the reaction solution of PBS $500 \mathrm{ml}$ and the reagent $50 \mathrm{ml}$, and reacted for $2 \mathrm{~h}$ in the incubator $\left(37^{\circ} \mathrm{C}, 5 \% \mathrm{CO} 2\right)$. Then, $550 \mathrm{ml}$ reaction solution was divided into $110 \mathrm{ml}$ in 96 well plates, and the light absorptions of the reaction solutions were measured by the plate reader (2030 ARVO ${ }^{\mathrm{TM}}$ X2, Perkin Elmer Co.) at a wavelength of $450 \mathrm{~nm}$. The cell number was then estimated from the resulting absorbance and the calibration curve of hMSC.

The gene expression of chondrogenesis was analyzed using the real-time polymerase chain reaction (PCR) reactions [16], [17]. Firstly, the samples after culture were washed with PBS and then frozen in liquid nitrogen $\left(-196^{\circ} \mathrm{C}\right)$. Each of the frozen samples was then crushed into powder in a mortar by a muddler while maintaining the frozen state by adding liquid nitrogen appropriately. The RNA was isolated from the powder from each samples using NucleoSpin®RNA II (MACHEREY-NAGEL GmbH \& Co.). The cDNA was synthesized by the reverse transcription polymerase chain reaction (RT-PCR) from the each RNAs applied the reagent (PrimeScript ${ }^{\circledR}$ RT Master Mix Perfect Real Time, Takara Bio Inc.). After addition of the reagent 
(SYBR $®$ Premix Ex Taq ${ }^{\text {TM }}$ II (Tli RNaseH Plus)) to synthesized cDNA, RT-PCR amplification was performed using the RT-PCR system (the thermal cycler Dice Real Time System TP960, Takara Bio Inc.). $\beta$-Actin was used as the housekeeping control and results were quantified for collagen type II and aggrecan using the $\Delta \Delta$ Ct relative quantification method [18], [19]. The primer pairs used in this study are listed in Table 1.

Table 1. Sequences of Primers Used for Real-Time PCR Analysis

\begin{tabular}{ll}
\hline \hline Genes & Sequences \\
\hline$\beta$-actin & 5'-TGGCACCCAGCACAATGAA-3' \\
& 5'-CTAAGTCATAGTCCGCCTAGAAGCA-3' \\
Collagen type II & 5'-GACTGGCGACACTTGCGTCTA-3' \\
& 5'-GGAGCCAGATTGTCATCTCCATA-3' \\
Aggrecan & 5'-CTGAACGACAGGACCATCGAA-3' \\
& 5'-CGTGCCAGATCATCACCACA-3' \\
\hline \hline
\end{tabular}

\subsection{Mechanical Characterization}

Compression tests were conducted periodically by using a conventional testing machine (EZ-Test, SHIMADZU Co.) at a loading rate of $1 \mathrm{~mm} / \mathrm{min}$ and room temperature after the cell cultures for $1,7,14,21$, and 28 days. The samples were under wet state by washing with PBS. Initial height and initial cross-sectional area of the samples were measured before compression test, and load-displacement relations were recorded during the compression test. The initial compressive modulus $(E)$ were then evaluated using the following formula:

$$
E=\frac{\sigma}{\varepsilon}=\frac{P L}{A \Delta L}
$$

where $\sigma$ is the stress, $\varepsilon$ the strain, $P$ the load under the compression test. $L$ is the height of the samples, and $\Delta L$ the displacement to the compression direction after loading at each time point.

\subsection{Microstructural Characterization}

The samples after culture were washed with PBS to remove the media, dehydrated with ethanol, immersed in t-butyl alcohol solution, and finally freeze-dried by a freeze-drying machine (ES-2030, Hitachi, Ltd.). Then, the freeze-dried scaffolds were mounted on aluminum stages by a carbon tape with the dotite and sputter-coated with Pt-Pd using an anion sputter coater (E-1030, Hitachi, Ltd.). Finally, the surfaces of samples were observed using a FE-SEM (S-4100, Hitachi, Ltd.) in order to characterize the proliferation behavior including the formation of ECM by hMSCs in the scaffolds.

\section{Results}

Variations of cell viability during each of control (no gel layer) and with gel layered are shown in Fig. 2. In both the two kinds of composite scaffolds, the cell number did not increase during the first half period of 14 days where the chondrogenic differentiation culture was performed. At 14 days of culture, the cell number of the control was about $1.8 \times 10^{5}$ cells/scaffold, while that of gel layered scaffold was about $1.4 \times 10^{5} \mathrm{cells} / \mathrm{scaffold}$. The cell number significantly increased during the second half period where the chondrocyte growth culture was performed. At 28 days of culture, the cell number of the control was about $16.5 \times 10^{5}$ cells/scaffold, while that of the gel layered was about $14.0 \times 10^{5}$ cells/scaffold. In this way, the gel layering tended to decrease the cell proliferation and this is thought to be due to rapid increase of fibroblast cells in the control.

The variations of the compressive modulus during culture are shown in Fig. 3. In both the composite 
scaffolds, the compression modulus did not change largely during the first half period with the chondrogenic differentiation culture. However, the modulus rapidly increased at 28 days of culture during the second half period with the chondrocyte growth culture. At 28 days of culture, the compression modulus of the control was about $6.0 \mathrm{kPa}$, and that of the gel layered was about $4.8 \mathrm{kPa}$. Thus, the gel layering tended to decrease the modulus due to the lower modulus of the gel layer and less proliferation behavior.

Variations of gene expression of type II collagen which is one of the typical proteins in cartilage tissue are shown in Fig. 4. In both the composite scaffold, type II collagen increased during the first half period with the chondrogenic differentiation culture, and kept almost constant during the second half period with the chondrocyte growth culture. The gel layering tended to increase the gene generation of type II collagen during culture. Variations of gene expression of aggrecan that is also one of the typical proteins in cartilage are shown in Fig. 5. In both cases, aggrecan significantly increased during the first half period. It also increased during the second half period with the chondrocyte growth culture. Thus, the gel layering tended to improve the gene formation of aggrecan during whole the 28 days of culture.

FE-SEM micrographs of surface of the control after 28 days culture are shown in Fig. 6. Many fibroblast-like flat cells were observed on the surface of the samples. FE-SEM micrographs of surface of the gel layered scaffold after 28 days culture are shown in Fig. 7. Many chondrocyte-like spherical cells and cotton-like fibrous type II collagen were observed on the surface of the samples. In comparison, more chondrocytes were observed in the gel layered scaffold than the control.

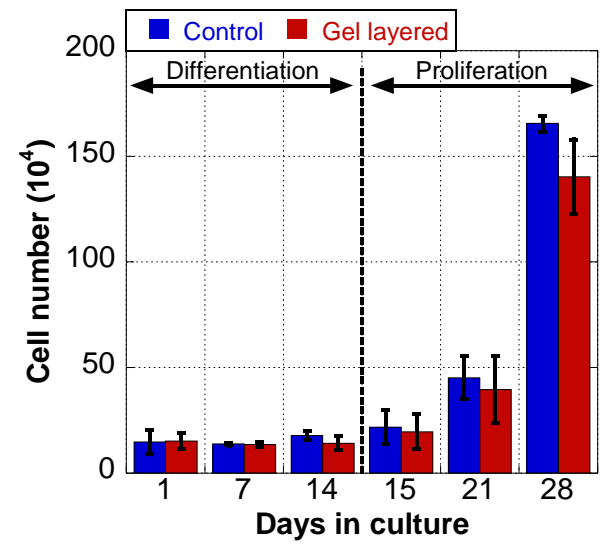

Fig. 2. Growth behavior of cells during culture.

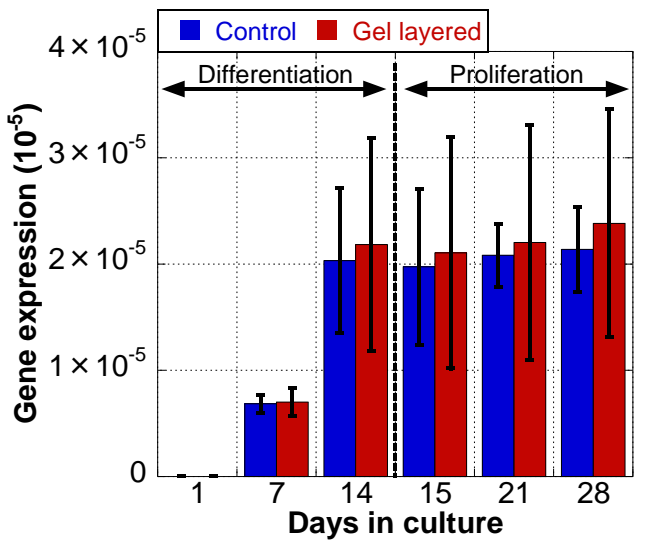

Fig. 4. Variation of type II collagen.

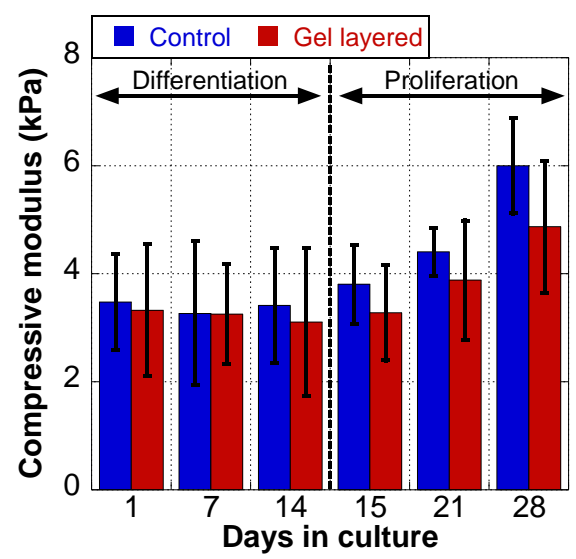

Fig. 3. Compressive modulus.

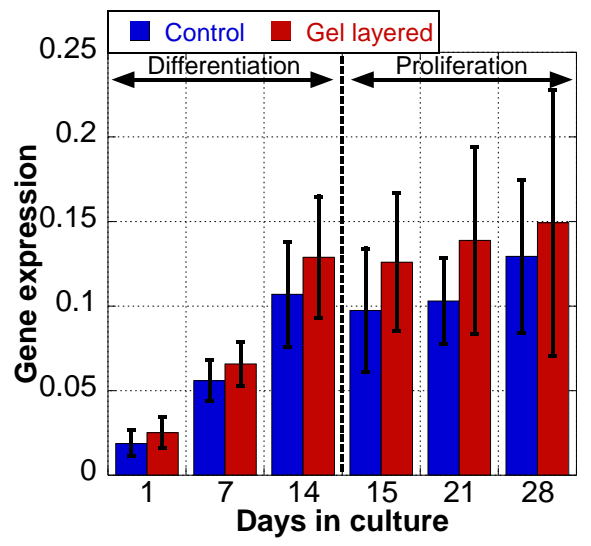

Fig. 5. Variation of aggrecan.

\section{Discussion}


In the combination culture, cell number did not change largely during the chondrogenic differentiation culture and increased during the cell growth culture as shown in Fig. 4. The cell number increased up to about 14 times in the gel layered scaffold and about 17 times in the control (the scaffold without gel layer) for 28 days culture. This suggests that the cell growth was suppressed by chondrogenic differentiation of hMSCs, and therefore the gel layer is effective for cartilage-like tissue formation.

In the combination culture, compressive modulus slightly decreased in the chondrogenic differentiation culture of the first half period, however it significantly increased in the chondrocyte growth culture of the second half period as shown in Fig. 3. It is thought that the structural stability was improved by hMSC proliferation and differentiation into chondrocytes, promotion of extracellular matrix formation and increase of formation amount, resulting in the recovery of the compressive modulus as shown in the FE-SEM micrograph of Figs. 6 and 7. The compression modulus at 28 days culture increased up to about 1.5 times in the gel layered scaffold and about 1.8 times in the control. The compression modulus of the gel layered at 28 days of culture was lower than that of the control, suggesting that the improvement of modulus of the gel layered was suppressed due to the suppression of cell growth by chondrogenic differentiation of hMSCs.

In both the scaffold, type II collagen did not increase in the cell proliferation culture, however it tended to increase with days in the chondrogenic differentiation culture as shown in Fig. 4. Increasing chondrogenic marker implies that hMSCs were differentiated into chondrocytes. The expression value of type II collagen of the gel layered scaffold was higher than that of the control during 28 days culture. It is thought that the gel layer is very effective for producing type II collagen. Also, gene expression of aggrecan by the gel layered was higher than that of the control as shown in Fig. 5, suggesting that the gel layering effectively activates cartilage-like tissue formation.

Many chondrocyte-like spherical cells were observed in the gel layered scaffold more than the control without gel layer as shown in Figs. 6 and 7. It implies that the gel layered scaffold activates chondrogenic differentiation of hMSCs, corresponding the because it corresponds to the chronological change of gene expression of type II collagen and aggrecan. This results clearly exhibited that the gel layer is very effective for differentiation of hMSCs into chondrocytes by introducing a suitable environment of three-dimensional floating positions for the cells.
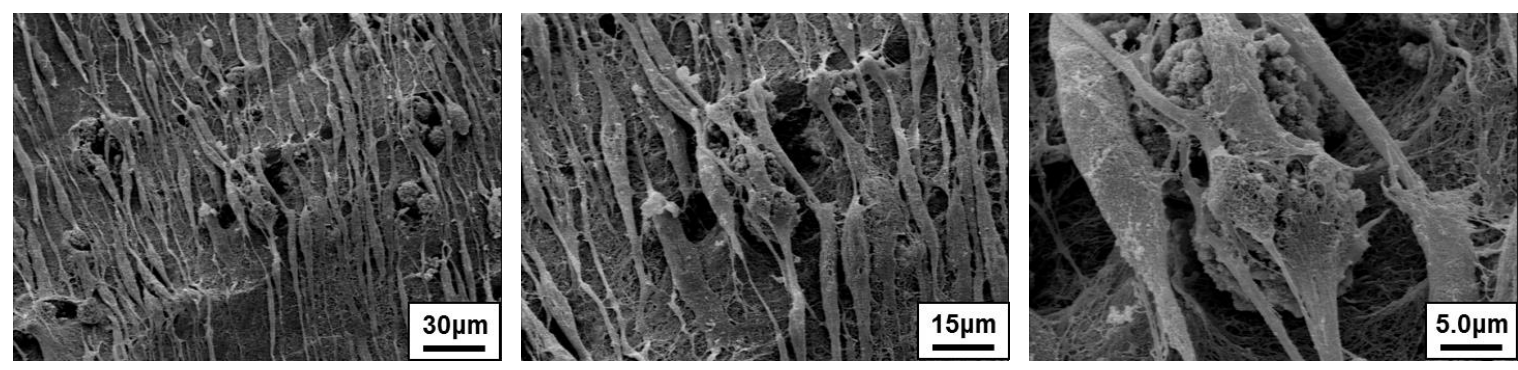

Fig. 6. FE-SEM micrographs of cells after 28 days (control).
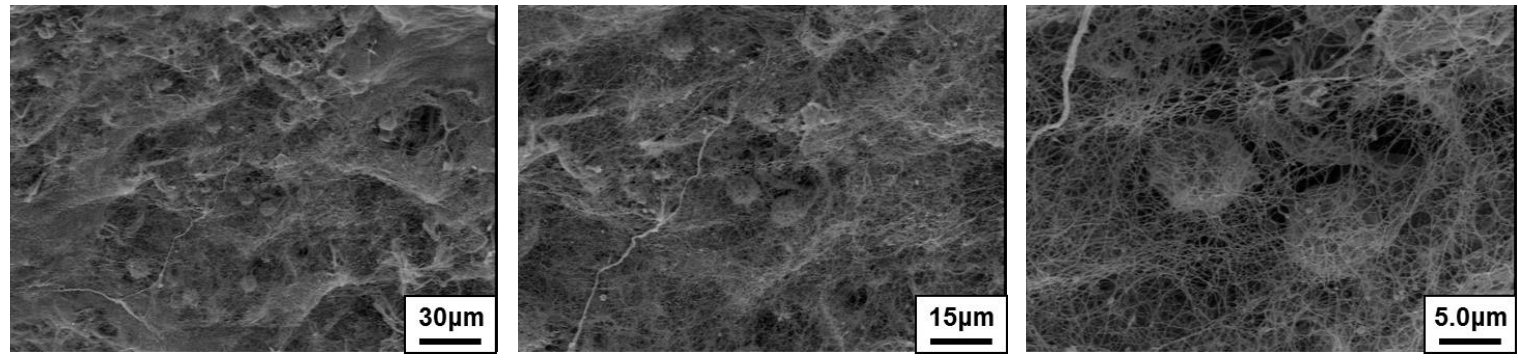

Fig. 7. FE-SEM micrographs of cells after 28 days (gel layered). 


\section{Conclusions}

In the present study, an innovative composite scaffold was developed for cartilage tissue engineering. The collagen sponge/gel composite scaffolds with hMSCs were wrapped by additional collagen gel layer. Culture experiments using the new scaffolds and the old ones (no gel layer) were performed and compared in order to characterize the effectiveness of the gel layering. The experimental results clearly exhibited that the introduction of gel layering is very effective for improvement of chondrogenic differentiation of hMSCs because the gel layering expressed large amount of gene expression of type II collagen and aggrecan which are typical chondrogenic markers, and furthermore many chondrocyte-like spherical cells with cotton-like type II collagen fibrils were found to be generated from FE-SEM observation. In the future study, we are planning to try to develop a tissue-engineered cartilage that has more similar structure with biological natural cartilage by examining the cell culture condition and the structure of the scaffold.

\section{Acknowledgement}

This work was supported by JSPS KAKENHI Grant Number JP15K13836.

\section{References}

[1] Lungu, E., Vendittoli, P. A., \& Desmeules, F. (2015). Identification of patients with suboptimal results after hip arthroplasty: Development of a preliminary prediction algorithm. BMC. Musculoskeletal. Disorders, 16, 279.

[2] Adachi, N., Ochi, M., Deie, M., Nakamae, A., Kamei, G., Uchio, Y., \& Iwasa, J. (2014). Implantation of tissue-engineered cartilage-like tissue for the treatment for full-thickness cartilage defects of the knee. Knee. Surg. Sports. Traumatol. Arthrosc, 22, 1241-1248.

[3] Liao, J., Qu, Y., Chu, B., Zhang, X., \& Qian, Z. (2015). Biodegradable CSMA/PECA/GRAPHENE porous hybrid scaffold for cartilage tissue engineering. Sci. Rep, 5, 9879.

[4] Ansboro, S., Hayes, J, S., Barron, V., Browne, S., Howard, L., Greiser, U., Lalor, P., Shannon, F., Barry, F. P., Pandit, A., \& Murphy, J. M. (2014). A chondromimetic microsphere for in situ spatially controlled chondrogenic differentiation of human mesenchymal stem cells. J. Control. Release, 179, 42-51.

[5] Jiang, Y., Jahagirdar, B. N., Reinhardt, R. L., Schwartz, R. E., Keene, C. D., \& Ortiz-Gonzalez, X. R., et al. (2002). Pluripotency of mesenchymal stem cells derived from adult marrow. Nature. Publishing. Group, 418, 41-49.

[6] Pittenger, M. F., Mackay, A. M., Beck, S. C., Jaiswal, R. K., Douglas, R., \& Mosca, J. D., et al. (1999). Multilineage potential of adult human mesenchymal stem cells. Science, 284, 143-147.

[7] Parmar, P. A., Chow, L. W., St-Pierre, J. P., Horejs, C. M., Peng, Y. Y., Werkmeister, J. A., Ramshaw, J. A., \& Stevens, M. M. (2015). Collagen-mimetic peptide-modifiable hydrogels for articular cartilage regeneration. Biomaterials, 54, 213-225.

[8] Guzzo, R. M., Scanlon, V., Sanjay, A., Xu, R. H., \& Drissi, H. (2014). Establishment of human cell type-specific iPS cells with enhanced chondrogenic potential. Stem. Cell. Rev, 10, 820-829.

[9] Iwasa, J., Ochi, M., Uchio, Y., Katsube, K., Adachi, N., \& Kawasaki, K. (2003). Effects of cell density on proliferation and matrix synthesis of chondrocytes embedded in atelocollagen gel. Artificial. Organs, 27, 249-255.

[10] Ma, Z., Gao, C., Gong, Y., \& Shen, J. (2005). Cartilage tissue engineering PLLA scaffold with surface immobilized collagen and basic fibroblast growth factor. Biomaterials, 26, 1253-1259.

[11] Meretoja, V.V., Dahlin, R. L., Wright, S., Kasper, F. K., \& Mikos, A. G. (2014). Articular chondrocyte redifferentiation in 3D co-cultures with mesenchymal stem cells. Tissue. Eng. Part C, 20, 514-523.

[12] Steinmetz, N. J., Aisenbrey, E. A., Westbrook, K. K., Qi, H. J., \& Bryant, S. J. (2015). Mechanical loading 
regulates human MSC differentiation in a multi-layer hydrogel for osteochondral tissue engineering. Acta. Biomaterialia, 21, 143-153.

[13] Rosenzweig, D. H., Carelli, E., Steffen, T., Jarzem, P., \& Haglund, L. (2015). 3D-Printed ABS and PLA scaffolds for cartilage and nucleus pulposus tissue regeneration. International. Journal. Molecular. Sciences, 16, 15118-115135.

[14] Yodmuang, S., McNamara, S. L., Nover, A. B., Mandal, B. B., Agarwal, M., \& Kelly, T. A., et al. (2015). Silk microfiber-reinforced silk hydrogel composites for functional cartilage tissue repair. Acta. Biomaterialia, 11, 27-36.

[15] Chen, G., Ushida, T., \& Tateishi, T. (2000). A biodegradable hybrid sponge nested with collagen microsponges. J. Biomed. Mater. Res, 51, 273-279.

[16] Hu, X., Zhu, J., Li, X., Zhang, X., Meng, \& Q., Yuan, L., et al. (2015). Dextran-coated fluorapatite crystals doped with $\mathrm{Yb} 3+/ \mathrm{Ho} 3+$ for labeling and tracking chondrogenic differentiation of bone marrow mesenchymal stem cells in vitro and in vivo. Biomaterials, 52, 441-451.

[17] Wang, Y., Kim, U. J., Blasioli, D. J., Kim, H. J., \& Kaplan, D. L. (2015). In vitro cartilage tissue engineering with 3D porous aqueous-derived silk scaffolds and mesenchymal stem cells. Biomaterials, 26, 7082-7094.

[18] Ding, X., Zhu, M., Xu, B., Zhang, J., Zhao, Y., \& Ji, S., et al. (2014). Integrated trilayered silk fibroin scaffold for osteochondral differentiation of adipose-derived stem cells. ACS. Appl. Mater. Interfaces, 6, 16696-16705.

[19] Wang, X., Li, Y., Han, R., He, C., Wang, G., \& Wang, J., et al. (2014). Demineralized bone matrix combined bone marrow mesenchymal stem cells, bone morphogenetic protein-2 and transforming growth factor- $\beta 3$ gene promoted pig cartilage defect repair. Plos. One, 9, e116061.

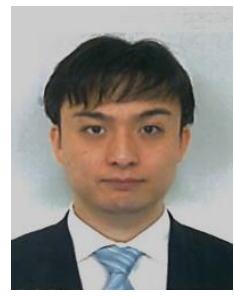

Yusuke Nakamuta received his bachelor and master's degrees from Kumamoto University in 2001 and Kyushu University in 2014, respectively. Currently he is a doctoral student of Kyushu University and working on cartilage tissue engineering

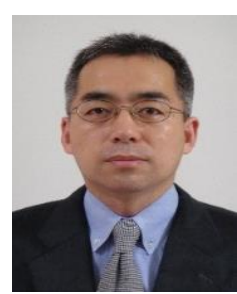

Mitsugu Todo received his master's and PhD degrees from Kyushu University in 1989 and the Ohio State University in 1995, respectively. He is currently an associate professor of Research Institute for Applied Mechanics, Kyushu University. His specializations are tissue engineering (bone, cartilage, blood vessel) using polymer and composite scaffolds, and computational biomechanics such as CT image based finite element analysis.

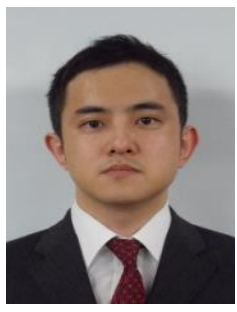

Takaaki Arahira received his master's and PhD degrees from Kyushu University in 2010 and in 2013, respectively. He is currently an assistant professor of Fukuoka Dental College. His specializations are tissue engineering (bone, cartilage) using organic-inorganic composite scaffolds with stem cells. 